\title{
Role of Urea in Intestinal Barrier Dysfunction and Disruption of Epithelial Tight Junction in Chronic Kidney Disease
}

\author{
Nosratola D. Vaziri ${ }^{a} \quad$ Jun Yuan ${ }^{a} \quad$ Keith Norris ${ }^{b}$ \\ ${ }^{a}$ Division of Nephrology and Hypertension, University of California, Irvine, Calif., and ${ }^{b}$ Department of \\ Internal Medicine, Charles Drew University, Los Angeles, Calif., USA
}

\section{Key Words}

Endotoxin • Inflammation - Gastrointestinal pathology •

End-stage renal disease $\cdot$ Uremia $\cdot$ Cardiovascular disease

\begin{abstract}
Background: Chronic kidney disease (CKD) impairs intestinal barrier function which leads to endotoxemia and systemic inflammation. We have found depletion of intestinal epithelial tight junction (TJ) proteins in animals with CKD. We further showed that addition of end-stage renal disease patients' plasma to the culture medium provokes a marked drop in transepithelial electrical resistance (TER) and depletion of TJ proteins in cultured human enterocytes. These effects were less severe with post- than with prehemodialysis plasma, suggesting a role of dialyzable agent(s). This study tested the hypothesis that intestinal barrier dysfunction in uremia may be due to diffusion of urea into the gut and its conversion to ammonia by microbial urease. Methods: Human enterocytes (T84 cells) were seeded on Transwell plates and utilized when TER exceeded $1,000 \mathrm{~m} \Omega \cdot \mathrm{cm}^{2}$ to ensure full polarization and TJ formation. Confluent cells were then incubated for $24 \mathrm{~h}$ in media containing 0,42 or $74 \mathrm{mg} / \mathrm{dl}$ urea or urea plus urease to simulate presence of microbial flora. Results: At clinically relevant concentrations, urea caused a concentration-dependent fall in TER and the key TJ proteins
\end{abstract}

claudin-1, occludin and zonula occludens 1. The effects of urea were dramatically amplified by urease causing cell detachment, dissipation of TER, and massive loss of TJ proteins. Conclusions: Uremia-induced disruption of intestinal $\mathrm{TJ}$ and barrier function is, in part, mediated by urea which is generally considered to be a nontoxic retained metabolite. These findings reveal a novel mechanism for a salutary effect of urea-lowering strategies, e.g. low-protein diet and longer and more frequent dialysis regimens in advanced CKD.

Copyright $\odot 2012$ S. Karger AG, Basel

\section{Introduction}

There is emerging evidence pointing to intestinal barrier dysfunction and its role in the pathogenesis of systemic inflammation in humans and animals with advanced chronic kidney disease (CKD) $[1,2]$. In an earlier study, we found marked depletion of the key transcellular (claudin-1 and occludin) and intracellular [zonula occludens 1 (ZO-1)] constituents of colonic epithelial tight junction (TJ) in the rats with CKD induced by either 5/6 nephrectomy or adenine-induced tubulointerstitial nephropathy. The reduction in the abundance of the TJ proteins was accompanied by their normal or even elevated mRNA abundance [3], suggesting a posttranscriptional or

\section{KARGER}

Fax +4161306 1234

E-Mail karger@karger.ch

www.karger.com (c) 2012 S. Karger AG, Basel

0250-8095/13/0371-0001\$38.00/0

Accessible online at:

www.karger.com/ajn
N.D. Vaziri, MD, MACP

Division of Nephrology and Hypertension, University of California Irvine Medical Center, Suite 400, City Tower, 101 The City Drive Orange, CA 92868 (USA)

E-Mailndvaziri@uci.edu 
posttranslational nature of the downregulation of these proteins. In a recent study [4], we asked whether uremiainduced intestinal epithelial barrier dysfunction and the $\mathrm{TJ}$ protein depletion are mediated by the retained uremic toxins/metabolites and if so, whether they can be removed by dialysis. To this end, we tested the effects of addition to the culture media of pre- and posthemodialysis plasma samples from end-stage renal disease (ESRD) patients and plasma from healthy controls on the polarized T84 human colonic epithelial cells. When seeded on Transwell plates, T84 cells form a polarized, impermeable monolayer that exhibits many of the functional characteristics of the intestinal epithelial cells in vivo, including vectorial solute transport and barrier function $[5,6]$. The study showed that compared with the control plasma, incubation in media containing predialysis plasma from ESRD patients caused a marked drop in transepithelial electrical resistance (TER) denoting increased epithelial permeability. This was accompanied by significant reductions in transcellular, i.e. claudin-1 and occludin, and intracellular, i.e. ZO-1, protein constituents of the epithelial TJ apparatus. The severity of TJ damage and dysfunction was significantly less in cells exposed to the postdialysis than predialysis plasma. These findings pointed to the presence of as yet unidentified, but partially dialyzable, product(s) in the uremic plasma capable of degrading epithelial TJ and impairing intestinal epithelial barrier function.

The reduction in urinary excretion of nitrogenous waste products in advanced CKD results in their accumulation in the body fluids. The most abundant among these metabolites is urea, which has been considered to be a nontoxic and inert retained uremic metabolite. The rise in urea concentration in the intracellular and extracellular fluid compartments results in its heavy influx into the gastrointestinal tract [7]. Within the intestinal lumen, urea is hydrolyzed by microbial urease forming large quantities of ammonia $\left[\mathrm{CO}\left(\mathrm{NH}_{2}\right) 2+\mathrm{H}_{2} \mathrm{O} \rightarrow \mathrm{CO}_{2}\right.$ $+2 \mathrm{NH}_{3}$ ] which is readily converted to ammonium hydroxide $\left[\mathrm{NH}_{3}+\mathrm{H}_{2} \mathrm{O} \rightarrow \mathrm{NH}_{4} \mathrm{OH}\right][8,9]$. Ammonium hydroxide, in turn, leads to a modest rise in the luminal fluid's $\mathrm{pH}$, causes mucosal irritation and promotes enterocolitis $[10,11]$. Based on these observations, we hypothesize that ammonium hydroxide generated from hydrolysis of urea by microbial urease in the intestinal lumen of uremic patients may contribute to the epithelial barrier dysfunction and erosion of the TJ protein constituents. The present study was designed to test this hypothesis by exploring the effect of clinically relevant concentrations of urea alone and urea plus urease on intestinal epithelial barrier function and structure in vitro.

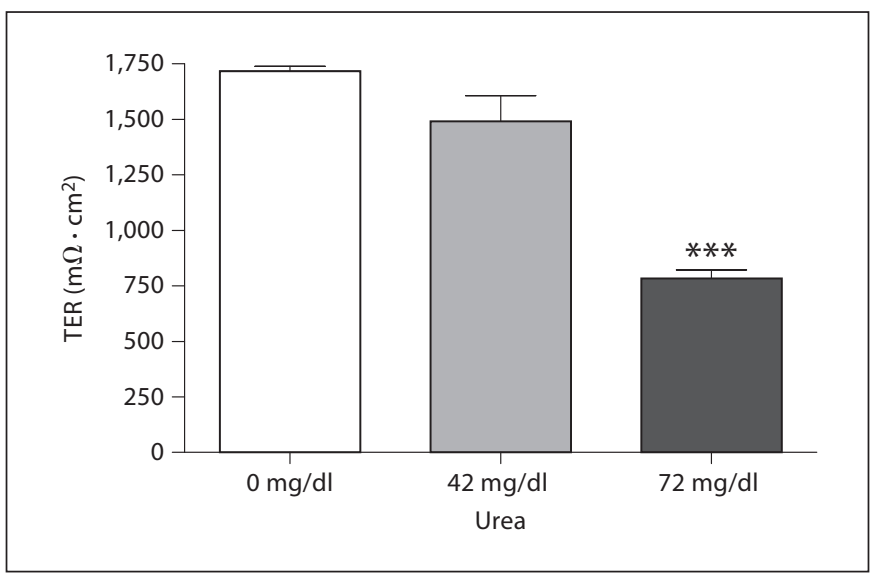

Fig. 1. Bar graphs depicting the TER in intestinal epithelial T84 cell monolayers incubated for $24 \mathrm{~h}$ in regular media and those incubated in media containing 42 or $72 \mathrm{mg} / \mathrm{dl}$ urea. ${ }^{* *} \mathrm{p}<0.001$.

\section{Methods}

Cell Culture and Incubation Studies

T84 cells were obtained from American Type Culture Collection (Manassas, Va., USA) and were grown in DMEM/F12 medium (Invitrogen Inc., Carlsbad, Calif., USA) containing sodium bicarbonate $1.2 \mathrm{~g} / \mathrm{l}$, L-glutamine $2.5 \mathrm{~mm}$, HEPES $15 \mathrm{~mm}$, and sodium pyruvate $0.5 \mathrm{~mm}$ (Invitrogen) supplemented with $10 \%$ fetal calf serum. To form polarized monolayers, cells were seeded $\left(60,000\right.$ cells $\left./ \mathrm{cm}^{2}\right)$ in 12-well or 6-well plates with Transwell inserts (Fisher Scientific, Pittsburgh, Pa., USA) for measurement of TER and Western blot analysis. The T84 monolayers were maintained for 21 days at $37^{\circ} \mathrm{C}$ in complete medium in an incubator containing a mixture of air with $5 \%$ carbon dioxide. The medium was changed every other day and the TER was measured regularly using Millicell ERS-2 meter (EMD Millipore Inc., Billerica, Mass., USA). When a TER exceeding $1,000 \mathrm{~m} \Omega \cdot \mathrm{cm}^{2}$ was reached, the monolayers were incubated for $24 \mathrm{~h}$ at $37^{\circ} \mathrm{C}$ in DMEM/F12 medium alone or in medium containing 42 or $72 \mathrm{mg} / \mathrm{dl}$ (70, or 120 $\mu \mathrm{M})$ urea (Sigma-Aldrich, St. Louis, Mo., USA) with or without urease (Sigma Inc.). The given urea concentrations were chosen to approximate the pre- and posthemodialysis values generally found in ESRD patients and as such were considered to be clinically relevant. Urease was added to the medium at 1 unit/ $\mu \mathrm{M}$ urea. At the conclusion of the 24-hour incubation period, the TER was measured and cells were harvested and processed for Western blot analysis.

\section{Western Blot Analyses}

The cells were lysed with M-PER Mammalian Protein Extraction Reagent (Fisher Scientific). Protein concentration in the cell lysate was determined by BSA assay kit (Pierce, Rockford, Ill., USA) and $20 \mu \mathrm{g}$ of total protein from each sample were fractionated on $4-12 \%$ Bis-Tris gradient gel (Invitrogen) at $120 \mathrm{~V}$ for $2 \mathrm{~h}$ and transferred to a nitrocellulose membrane. The membrane was then incubated with rabbit anti-claudin-1 or rabbit anti-occludin or mouse anti-ZO-1 (Invitrogen) antibodies at 1:250 dilu- 


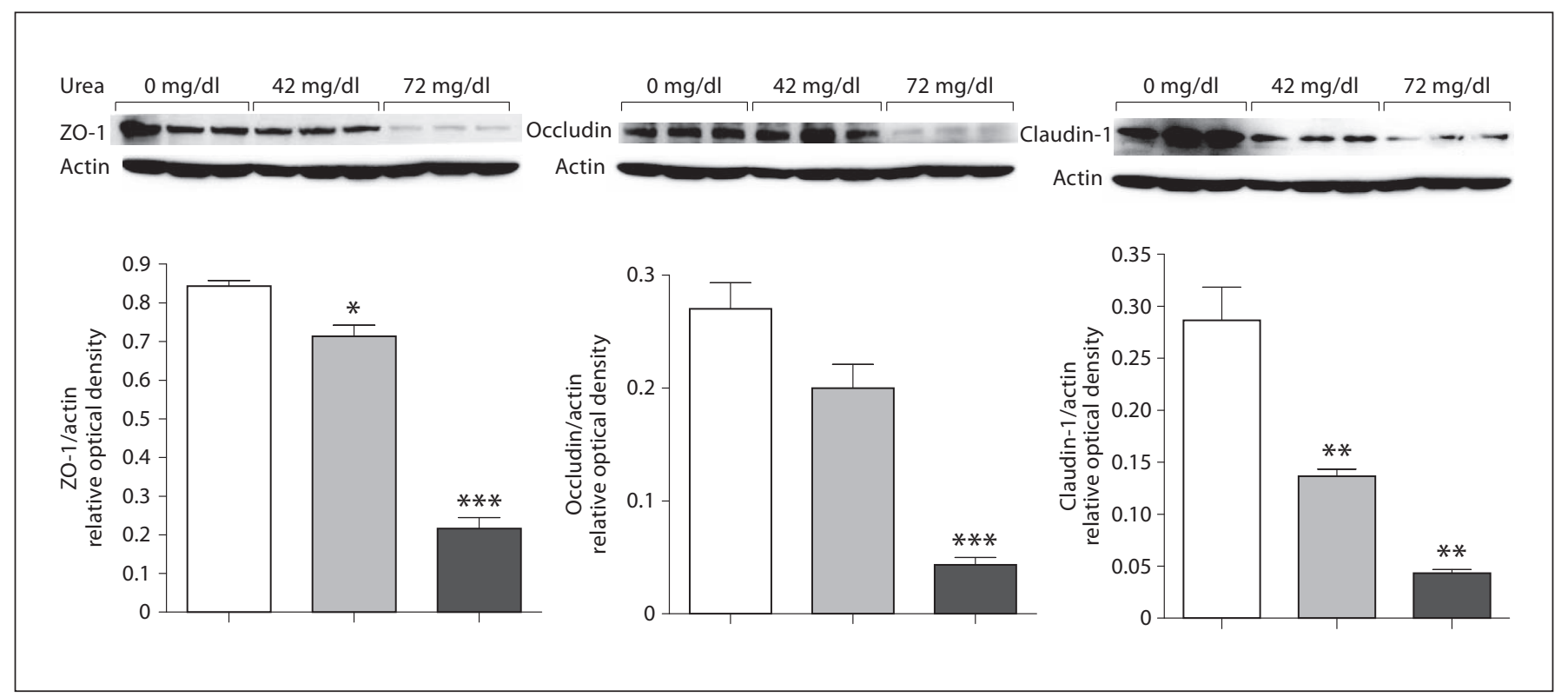

Fig. 2. Representative Western blots and group data depicting protein abundance of occludin, claudin-1 and ZO-1 in intestinal epithelial T84 cell monolayers incubated for $24 \mathrm{~h}$ in regular media and media containing 42 or $72 \mathrm{mg} / \mathrm{dl}$ urea. CTL $=$ Control. ${ }^{*} \mathrm{p}<0.05 ;{ }^{* *} \mathrm{p}<0.01 ;{ }^{* * *} \mathrm{p}<0.001$ versus control.

tions, and anti-actin antibody (Sigma-Aldrich) at a 1:10,000 dilution overnight. The appropriate horseradish peroxidase-conjugated secondary antibodies (Sigma-Aldrich) were used at a 1:5,000 dilution. The membrane was visualized with SuperSignal West Pico (Pierce) and developed by autoluminography.

\section{Data Analysis}

Analysis of variance and Student's t test were used in the statistical evaluation of the data which are presented as mean \pm SD. $\mathrm{p}$ values less than 0.05 were considered significant.

\section{Results}

\section{Effect of Urea and Urea plus Urease on TER and $p H$}

Incubation in media containing urea at 42 or $72 \mathrm{mg} / \mathrm{dl}$ resulted in a significant concentration-dependent fall in basal-apical electrical resistance pointing to the ability of urea to impair epithelial barrier function (fig. 1). Epithelial monolayers incubated in media containing both urea and urease exhibited detachment which precluded the ability to measure TER. Addition of urease alone to the culture medium had no significant effect on TER. The average $\mathrm{pH}$ of the culture medium rose from 7.1 to 7.3, $24 \mathrm{~h}$ after addition of urea plus urease.

\section{Effect of Urea and Urea plus Urease on Epithelial TJ Proteins}

The fall in the monolayer TER in cells incubated in urea-containing media was associated with significant concentration-dependent reductions in claudin-1, occludin, and ZO-1 abundance (fig. 2, 3). Addition of urease to the incubation media designed to simulate the presence of urease-possessing colonic bacteria led to a further and drastic fall in claudin-1, occludin and ZO-1 abundance (fig. 4).

\section{Discussion}

Systemic inflammation is a constant feature and a major mediator of cardiovascular disease, cachexia, anemia and numerous other morbidities in patients with advanced CKD [12-16]. Inflammation in these patients is commonly associated with endotoxemia in the absence of clinical infection [17-19]. The most likely source of endotoxemia in the infection-free patients is the gut microbial flora. However, entry of endotoxin from the intestinal lumen to the circulation can occur only when the mucosal barrier is impaired. As summarized in recent reviews [1, 2], there is compelling evidence supporting the impairment of the intestinal barrier function and its potential 


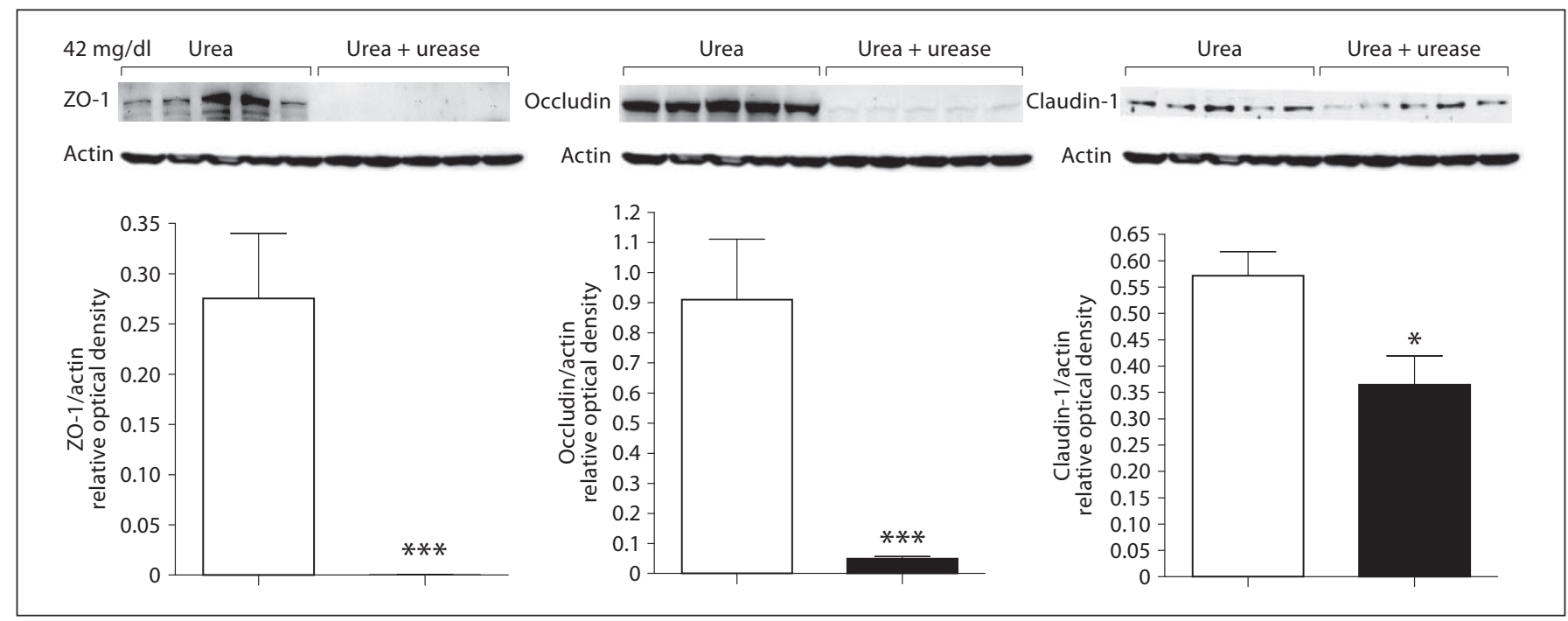

Fig. 3. Representative Western blots and group data depicting protein abundance of occludin, claudin-1 and ZO-1 in intestinal epithelial T84 cell monolayers incubated for $24 \mathrm{~h}$ in media containing $42 \mathrm{mg} / \mathrm{dl}$ urea alone and those incubated in media containing $42 \mathrm{mg} / \mathrm{dl}$ urea plus urease. ${ }^{*} \mathrm{p}<0.05 ;{ }^{* *} \mathrm{p}<0.0001$ versus control.

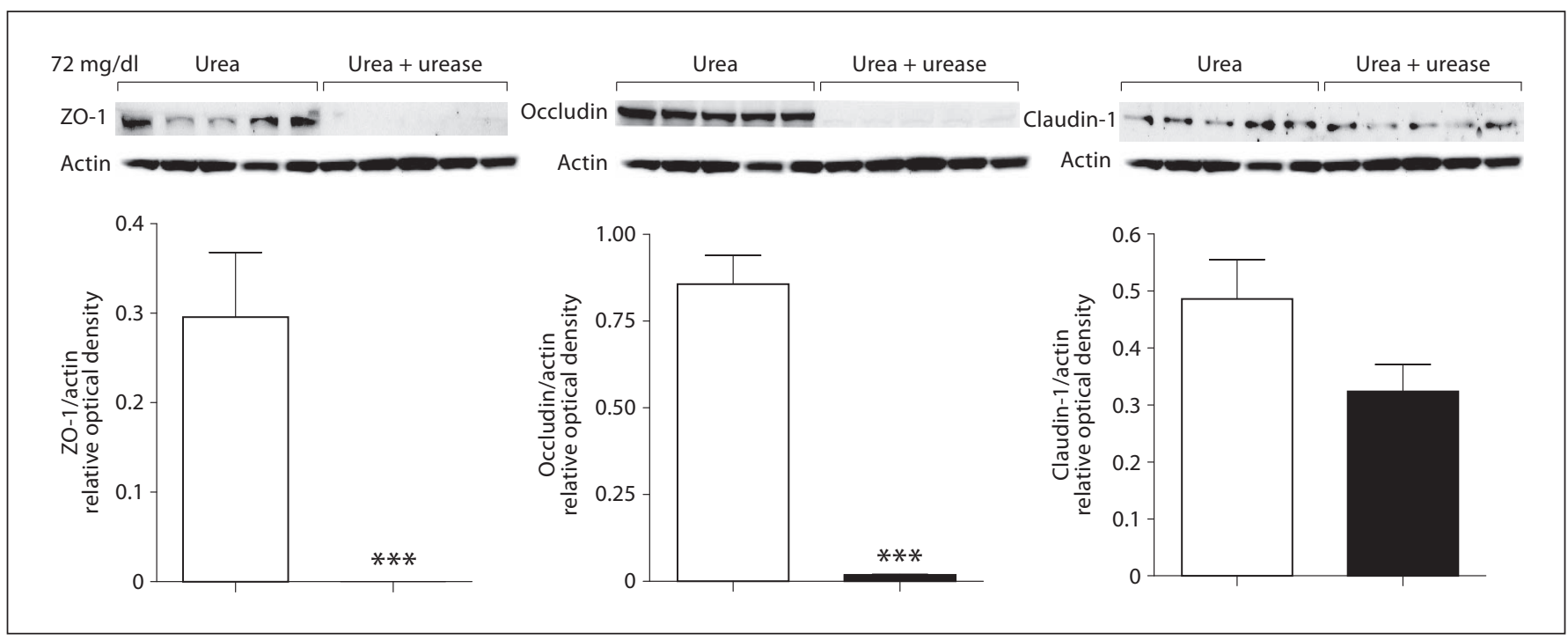

Fig. 4. Representative Western blots and group data depicting protein abundance of occludin, claudin-1 and ZO-1 in intestinal epithelial T84 cell monolayers incubated for $24 \mathrm{~h}$ in media containing $72 \mathrm{mg} / \mathrm{dl}$ urea alone and those incubated in media containing $72 \mathrm{mg} / \mathrm{dl}$ urea plus urease. ${ }^{* *} \mathrm{p}<0.001$ versus control.

contribution to the prevailing inflammation in the uremic humans. This is based on the reported increase in intestinal permeability to high-molecular-weight polyethylene glycols $[17,20]$, presence of endotoxemia [17, 19], and histological evidence of chronic inflammation throughout the gastrointestinal tract in this population $[10,11]$. The TJ apparatus is essential for the epithelial polarity and restriction of paracellular permeability of intestinal epithelium. Consequently, disruption of the intestinal epithelial TJ can facilitate the influx of noxious 
microbial and other luminal contents in the intestinal wall and their subsequent release throughout the body via the vascular and lymphatic channels. This can, in turn, lead to local and systemic inflammation and accumulation of uremic toxins generated by the gut microbiota.

The present study revealed that at clinically relevant concentrations, urea significantly lowered the TER in the TJ-forming polarized human colonic epithelial cell monolayer in vitro. The effect of urea was dramatically amplified by urease, which was used to simulate the effect of urease-possessing microbial species in the colon. These observations illustrate the potential contribution of influx of urea into the intestinal tract in the pathogenesis of the uremia-induced intestinal barrier dysfunction in the uremic patients. The observed fall in the TER was accompanied by and was largely due to the urea concentration-dependent depletion of occludin, claudin-1, and ZO-1 which are the key protein constituents of the epithelial TJ. The erosive effect of urea on the measured TJ proteins was greatly intensified in the presence of urease leading to near-complete depletion of ZO-1 and occludin and severe reduction in claudin-1 abundance. As noted above, accumulation of urea in the intra- and extracellular fluid compartments in patients and animals with advanced CKD results in its heavy influx into the gastrointestinal tract via passive diffusion and incorporation in the glandular secretions [7-9]. As noted earlier, within the intestinal lumen urea is hydrolyzed spontaneously and by microbial urease forming large quantities of ammonia $[8,9]$. Ammonia is, in turn, converted to ammonium hydroxide which is a caustic base capable of causing cytotoxicity and tissue damage. Recent studies conducted by our group have shown marked changes in the composition of microbial flora in humans and animals with advanced CKD [21]. Five of the 10 microbial families which were most abundant in the CKD patients possessed functional urease $[2,21]$. Thus massive influx of urea into the gastrointestinal tract together with the dominance of urease-possessing bacteria in the uremic individuals work in concert to promote formation of ammonia and ammonium hydroxide in the intestinal tract $[8,9]$. This can account for the dramatic impairment of the barrier function and destruction of TJ apparatus observed in the present in vitro experiments designed to simulate the effect of uremia in vivo.

In conclusion, the CKD-induced disruption of intestinal TJ and impairment of the gut barrier function is, in part, mediated by urea which heretofore was considered to be a nontoxic uremic retained metabolite. These findings illustrate the adverse effect of the high urea levels and reveal a novel mechanism for the salutary effect of urealowering strategies such as low-protein diet and longer and more frequent dialysis regimens in patients with advanced CKD.

\section{Acknowledgements}

This study was in part funded by the NIH grants RR026138 and MD000182.

\section{Disclosure Statement}

The authors have no conflict of interest.

\section{References}

$>1$ Ritz E: Intestinal-renal syndrome: mirage or reality? Blood Purif 2011;31:70-76.

$\checkmark 2$ Vaziri ND: CKD impairs barrier function and alters microbial flora of the intestine - a major link to inflammation and uremic toxicity. Curr Opin Nephrol Hypertens 2012;21: 587-592.

$>3$ Vaziri ND, Yuan J, Rahimi A, Ni Z, Said H, Subramanian VS: Disintegration of colonic epithelial tight junction in uremia: a likely cause of CKD-associated inflammation. Nephrol Dial Transplant 2012;27:26862693.
-4 Vaziri ND, Goshtasby N, Yuan J, Jellbauer S, Moradi H, Raffatellu M, Kalantar-Zadeh K: Uremic plasma impairs barrier function and depletes the tight junction protein constituents of intestinal epithelium. Am J Nephrol 2012;36:438-443.

5 Madara JL, Dharmsathaphorn K: Occluding junction structure-function relationships in a cultured epithelial monolayer. J Cell Biol 1985;101:2124-2133.

6 Dharmsathaphorn K, McRoberts JA, Mandel KG, Tisdale LD, Masui H: A human colonic tumor cell line that maintains vectoral electrolyte transport. Am J Physiol 1984; 246:G204-G208
7 Lee YT: Urea concentration in intestinal fluids in normal and uremic dogs. J Surg Oncol 1971;3:163-168.

-8 Bourke E, Milne MD, Stokes GS: Caecal pH and ammonia in experimental uraemia. Gut 1966;7:558-561.

$\checkmark 9$ Swales JD, Tange JD, Evans DJ: Intestinal ammonia in uraemia: the effect of a urease inhibitor, acetohydroxamic acid. Clin Sci 1972;42:105-112.

10 Kang JY: The gastrointestinal tract in uremia. Dig Dis Sci 1993;38:257-268. 
11 Vaziri ND, Dure-Smith B, Miller R, et al: Pathology of gastrointestinal tract in chronic hemodialysis patients: an autopsy study of 78 cases. Am J Gastroenterol 1985;80:608-611.

12 Vaziri ND, Pahl MV, Crum A, Norris K: Effect of uremia on structure and function of immune system. J Ren Nutr 2012;22:149156.

13 Carrero JJ, Stenvinkel P: Inflammation in end-stage renal disease - what have we learned in 10 years? Semin Dial 2010;23:498509.

14 Himmelfarb J, Stenvinkel P, Ikizler TA, et al: The elephant in uremia: oxidant stress as a unifying concept of cardiovascular disease in uremia. Kidney Int 2002;62:1524-1538.
15 Yoon JW, Pahl MV, Vaziri ND: Spontaneous leukocyte activation and oxygen-free radical generation in end stage renal disease. Kidney Int 2007;71:167-172.

16 Gollapudi P, Yoon J-W, Gollapudi S, et al: Effect of end stage renal disease and hemodialysis on expression and activities of leukocyte toll-like receptors (TLR). Am J Nephrol 2010;31:247-254.

17 Feroze U, Kalantar-Zadeh K, Sterling KA, Molnar MZ, Noori N, Benner D, Shah V, Dwivedi R, Becker K, Kovesdy CP, Raj DS: Examining associations of circulating endotoxin with nutritional status, inflammation, and mortality in hemodialysis patients. J Ren Nutr 2012;22:317-326.

18 Gonçalves S, Pecoits-Filho R, Perreto S, et al: Associations between renal function, volume status and endotoxaemia in chronic kidney disease patients. Nephrol Dial Transplant 2006;21:2788-2794.
19 Szeto CC, Kwan BC, Chow KM, et al: Endotoxemia is related to systemic inflammation and atherosclerosis in peritoneal dialysis patients. Clin J Am Soc Nephrol 2008;3:431436.

20 Magnusson M, Magnusson KE, Sundqvist T, et al: Impaired intestinal barrier function measured by differently sized polyethylene glycols in patients with chronic renal failure. Gut 1991;32:754-759.

21 Vaziri ND, Wong J, Pahl MV, Piceno YM, Yuan J, DeSantis TZ, Ni Z, Nguyen TH, Andersen GL: Chronic kidney disease alters the composition of intestinal microbial flora. Kidney Int 2012, DOI: 10.1038/ki.2012.345 E-pub ahead of print. 